\title{
Acceleration of the Particle Swarm Optimization for Peierls-Nabarro modeling of dislocations in conventional and high-entropy alloys th
}

\author{
Zongrui Pei ${ }^{1}$, \\ Oak Ridge National Laboratory, Oak Ridge, TN 37831, USA; Max-Planck-Institut für Eisenforschung GmbH,40237, Düseldorf, Germany \\ Markus Eisenbach \\ Oak Ridge National Laboratory, Oak Ridge, TN 37831, USA
}

\begin{abstract}
Dislocations are among the most important defects in determining the mechanical properties of both conventional alloys and highentropy alloys. The Peierls-Nabarro model supplies an efficient pathway to their geometries and mobility. The difficulty in solving the integro-differential Peierls-Nabarro equation is how to effectively avoid the local minima in the energy landscape of a dislocation core. Among the other methods to optimize the dislocation core structures, we choose the algorithm of Particle Swarm Optimization, an algorithm that simulates the social behaviors of organisms. By employing more particles (bigger swarm) and more iterative steps (allowing them to explore for longer time), the local minima can be effectively avoided. But this would require more computational cost. The advantage of this algorithm is that it is readily parallelized in modern high computing architecture. We demonstrate the performance of our parallelized algorithm scales linearly with the number of employed cores.
\end{abstract}

Keywords: Peierls-Nabarro model, dislocations, Particle Swarm Optimization, acceleration

\section{Introduction}

Dislocations are one of the most important classes of defects that dominate the mechanical properties of crystalline solids [1, 2]. Since it was proposed to explain the huge discrepancy 5 between theoretically predicted and experimentally measured resolved shear stresses of materials, the primary pathway to study dislocations had been experimental methods. With the advance of computing and computational sciences, theoretical simulations of dislocations have emerged as a complemen-

10 tary approach to systematic crystal mechanical experiments to gain insight into the deformation mechanisms [3, 4, 5, 6, 7]. Additionally, when the knowledge of atomic-scale dislocation core structures are critical, theoretical simulations are particularly useful [5, 8, 9, 10]. As examples, Yasi et al. [5] simulated the core structures of basal and prismatic dislocations in $\mathrm{Mg}$ using density functional theory (DFT). Later the same authors developed different models to calculate the critical resolved shear stresses (CRSS) of basal dislocations in $\mathrm{Mg}$ al-

\footnotetext{
This manuscript has been authored by UT-Battelle, LLC under Contract No. DE-AC05-00OR22725 with the U.S. Department of Energy. The United 40 States Government retains and the publisher, by accepting the article for publication, acknowledges that the United States Government retains a nonexclusive, paid-up, irrevocable, world-wide license to publish or reproduce the published form of this manuscript, or allow others to do so, for United States Government purposes. The Department of Energy will provide public access to these results of federally sponsored research in accordance with the DOE Public Access Plan (http://energy.gov/downloads/doe-public-access-plan).

Email address: peizongrui@gmail.com (Zongrui Pei )

${ }^{1}$ Corresponding author
}

loys [11] and the cross-slip stresses of prismatic screw dislocations in $\mathrm{Mg}$ [12]. As another example, Shin and Carter applied orbital-free density functional theory (OFDFT) method to model dislocation core structures [6] and subsequently also calculated Peierls stresses in pure $\mathrm{Mg}$ [10].

The common feature of the aforementioned atomic-scale simulations is that dislocation cores are described explicitly in an atom-by-atom manner. We refer the methods employed as direct methods. Within this group of methods, the dislocation core structures are relaxed by molecular statics/dynamics simulations using either empirical embedded-atom-method (EAM) potentials [3] or DFT [13, 14]. While the DFT based methods are accurate they are computationally very expensive for simulating dislocations that require usually several hundreds of atoms or even many more due to their long-range fields. Atomistic simulations are efficient but very often there are no reliable EAM potentials for the materials systems of interest.

In contrast to the direct methods, indirect methods do not describe dislocation core structures explicitly by using misfit functions (denoted by $u$ in this work). A misfit function quantifies the distribution of the Burgers vector of a dislocation in space coordinate, i.e., the geometry of a dislocation core. It can be obtained from the Peierls-Nabarro model that was proposed by Peierls and Nabarro [15, 16, 17] about seventy years ago. The original model is applicable for one-dimensional dislocation cores. Later, Leibfried and Dietze generalized the onedimensional model to two-dimensional case that can address dissociated planar dislocation cores [18]. The primary input is taken from the generalized stacking fault energies (GSFEs, 


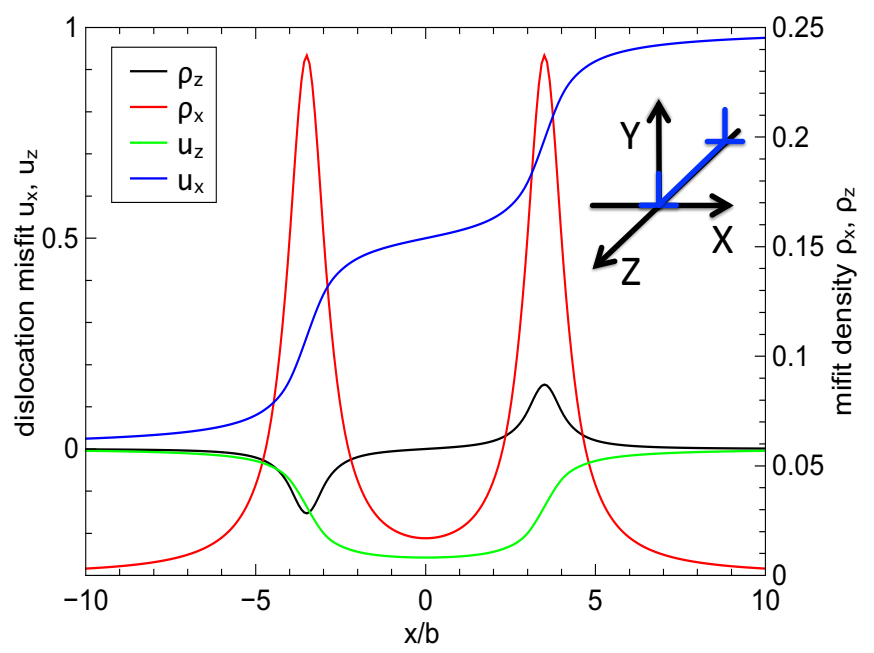

Figure 1: The two components $\left(u_{x}, u_{z}\right)$ of the misfit function of basal $\langle a\rangle$ dislocation in $\mathrm{Mg}$ and their derivatives $\left(\rho_{x}, \rho_{z}\right)$. The dislocation is placed in a coordinate as shown in the inset, i.e., the dislocation line runs along $z$ axis, and the slip plane is $x z$ plane. The $z$ axis is perpendicular to the paper.

so-called gamma surface) proposed by Vítek [19]. The gamma surface can be accurately calculated by DFT methods with computationally available and acceptable cost. Therefore, PeierlsNabarro modeling represents an alternative to the above dis- 90 cussed direct fully atomistic simulation methods for dislocations with planar core structures. In the last years these models have been substantially improved and optimized. For example, Wang added gradients of the misfit density to the total energy functional of dislocations in order to address dislocations possessing narrow cores [20]. The misfit density refers to the first derivative of misfit function with respective to a space variable, which will be discussed in more detail below. Bulatov et al. [21] discretized the misfit density and employed an array of discrete data points to describe it (for an application example in $\mathrm{Al}$, see [22]), since the discretized functions can provide more flexibility than the analytic (arctan-type) functions.

The choice of a suitable variant of Peierls-Nabarro model (i.e., a suitable form for the trial solution) depends on the specific problem to be addressed. For example, the dislocation core structures in $\mathrm{Mg}$ are usually very complicated, but the basal dislocation has a dissociated planar core, which can be adequately described by four actan functions using the stan70 dard two-dimensional Peierls-Nabarro model [7]. For dislocations with significantly narrower cores, more arctan functions are usually required.

\section{Peierls-Nabarro Model}

In the framework of classical Peierls-Nabarro model, the equilibrium configuration of a dislocation is determined by the balance of restoring force and elastic resistance of the crystal lattice. Here we assume an edge dislocation aligned with the Cartesian coordinate system. The dislocation line goes along the $z$ axis (for screw dislocation the dislocation line runs along the $x$ axis) and its Burgers vector $\vec{b}$ is parallel to $x$ (see Figure
1). Such a dislocation is described mathematically by

$$
2 K \int_{-\infty}^{+\infty} \frac{\vec{\rho}\left(x^{\prime}\right)}{x-x^{\prime}} \mathrm{d} x^{\prime}=-\frac{\mathrm{d} \gamma(\vec{u})}{\mathrm{d} \vec{u}}
$$

where the left side is the elastic resistance and the right side represents the restoring forces. Specifically $\vec{u}$ is the misfit function and $\vec{\rho}(x)$ is the misfit density, $\vec{\rho}(x)=\mathrm{d} \vec{u}(x) / \mathrm{d} x$. The generalized stacking fault energy, $\gamma(\vec{u})$, or gamma surface (see Section 2.1 , is the key input quantity of the model. In the two dimensional case, the misfit function $\vec{u}$ is a two-dimensional vector consisting of components along $x$ and $z$, i.e., $u_{x}$ and $u_{z}$ respectively. Further, the energy factor $K$ is a proportionality that is in elastically isotropic materials related to the shear modulus $\mu$ and the Poisson ratio $v . K=K_{e}=\mu / 4 \pi(1-v)$ for edge dislocations and $K=K_{s}=\mu / 4 \pi$ for screw dislocations.

55 The subscripts "e" and "s" in $K_{e}$ and $K_{s}$ indicate whether the constant is for an edge or a screw dislocation. In elastically anisotropic media, $K$ depends on the slip system and dislocation character and is calculated from elastic constants. For a basal $\langle a\rangle$ edge/screw dislocation in hexagonal close-packed materials,$K_{e}=1 / 4 \pi\left(\bar{C}_{11}+C_{13}\right)\left(\left(C_{44}\left(\bar{C}_{11}-C_{13}\right)\right) /\left(C_{33}\left(\bar{C}_{11}+\right.\right.\right.$ $\left.\left.\left.C_{13}+2 C_{44}\right)\right)\right)^{1 / 2}, \bar{C}_{11}=\left(C_{11} C_{33}\right)^{1 / 2} ; K_{s}=1 / 4 \pi\left(C_{44} C_{66}\right)^{1 / 2}$, $C_{66}=\left(C_{11}-C_{12}\right) / 2[1]$.

An analytical solution of $\vec{u}$ determined by Eq. 1 exists only for rather simple gamma surfaces. When a gamma surface is described by one cosine function, the exact solution is one arctan function. A dislocation described by one arctan-type function is called Peierls dislocation. For more complex (and more realistic) gamma surfaces, solutions can be obtained only numerically. Often, the solution is obtained by expanding $\vec{u}$ into a series of arctan functions [17]. The arctan function is a suitable basis since it captures the main characters of a dislocation. As an example for an edge dislocation of Burgers vector $\vec{b}$, the misfit function $\vec{u}=\left(u_{e}, u_{s}\right)$,

$$
\begin{aligned}
& u_{e}=\frac{b}{\pi} \sum_{i} A_{i} \arctan \left(\frac{x-d_{e, i}}{w_{e, i}}\right)+\frac{b}{2}, \quad \sum_{i} A_{i}=1 ; \\
& u_{s}=\frac{b}{\pi} \sum_{i} B_{i} \arctan \left(\frac{x-d_{s, i}}{w_{s, i}}\right), \quad \sum_{i} B_{i}=0 .
\end{aligned}
$$

where $A_{i}, B_{i}$ determine the weights of Peierls dislocations, $d_{e, i}, d_{s, i}$ and $w_{e, i}, w_{s, i}$ characterize the position and half-width of the Peierls dislocations. The subscript differentiates the edge component from the screw component.

By proposing the solution in the form of trial functions as suggested in Eq. 2 and applying the variational principle we transform the integral-differential Eq. 1 into an optimization problem. The left part of Eq. 1 is transformed into the elastic 
Table 1: (Algorithm 1) The self-consistent procedure to solve Peierls-Nabarro equation.

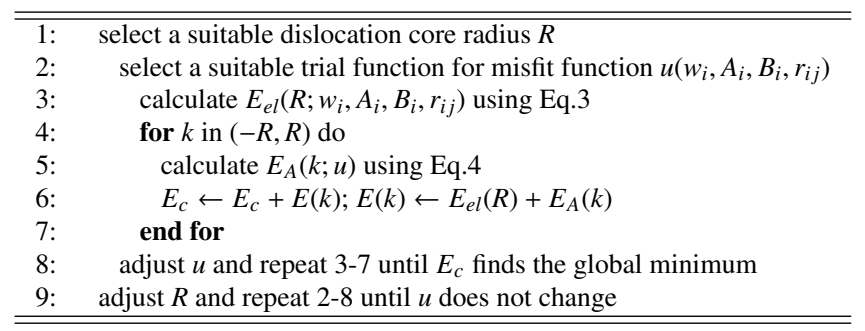

energy $E_{e l}[17]$,

$E_{e l}=H_{11}\left(\sum_{i, j} A_{i} A_{j} \ln \left(\frac{R}{w_{e, i}+w_{e, j}}\right)-\sum_{i<j} A_{i} A_{j} \ln \left(1+\frac{r_{e, i j}^{2}}{\left(w_{e, i}+w_{e, j}\right)^{2}}\right)\right)$ $\left.+H_{33}\left(\sum_{i, j} B_{i} B_{j} \ln \left(\frac{R}{w_{s, i}+w_{s, j}}\right)\right)-\sum_{i<j} B_{i} B_{j} \ln \left(1+\frac{r_{s, i j}^{2}}{\left(w_{s, i}+w_{s, j}\right)^{2}}\right)\right) \begin{aligned} & \text { Figure 2: Convergence of the Peierls stress of the <a }>\text {-type edge dislocation in } \\ & \text { pure Mg and Mg-Y as a function of the plane wave cutoff [7]. }\end{aligned}$ $+2 H_{13}\left(\sum_{i, j} A_{i} B_{j} \ln \left(\frac{R}{w_{e, i}+w_{s, j}}\right)-\frac{1}{2} \sum_{i, j} A_{i} B_{j} \ln \left(1+\frac{r_{i j}^{2}}{\left(w_{e, i}+w_{s, j}\right)^{25}}\right)\right)$

In this expression $\mathrm{H}$ is the Stroh tensor. The terms in the first bracket represent the elastic energy of the edge component, the second stands for the elastic energy of the screw component. $\left\{H_{11}, H_{33}\right\}=\left\{K_{e}, K_{s}\right\}$. The last two terms in Eq. 3 vanish when the dislocation core structure is symmetric, which is actually the cases for basal dislocations in hep or $\{111\}<1 \overline{1} 0>$ dislocations in face-centered cubic (fcc) crystalline solids. $R$ is the outer cutoff radius, $r_{i j}$ is the distance between edge component of Peierls dislocation $j$ and screw component of Peierls dislocation $i$, and $r_{e, i j}$ or $r_{s, i j}$ is the distance between the edge or screw components of the Peierls dislocations $i$ and $j$.

The right part is transformed into the misfit energy $E_{A}$, i.e., the integral of the gamma surface within the core region of a dislocation

$$
E_{A}=\int_{-R}^{R} \gamma\left(u_{x}(x), u_{z}(x)\right) d x .
$$

The Peierls stress $\tau_{p}$ is taken as the maximum of the gradient of the misfit energy $E_{A}$, i.e., $\tau_{p}=\max \left\{\frac{d E_{A}}{d u}\right\}$. Since the stress field far away from the dislocation core does not affect the core structure, a large but finite $R$ is sufficient for the evaluation. A larger $R$ does not change the numerical solutions. The total energy of a dislocation is the sum of the misfit energy and the elastic energy,

$$
E_{t o t}=E_{e l}+E_{A} .
$$

Minimizing the total energy $E_{\text {tot }}$ to create a dislocation is equivalent to solve Eq. 1] Minimization of $E_{t o t}$ (Eq. 5) yields the geometry (characterized by the parameters $w$ and $d$ ) of the studied dislocations. We summarize the self-consistent procedure to solve the Peierls-Nabarro equation in Table 1 For conducting the minimization we use the algorithm of Particle Swarm Optimization, since this algorithm can effectively avoid local

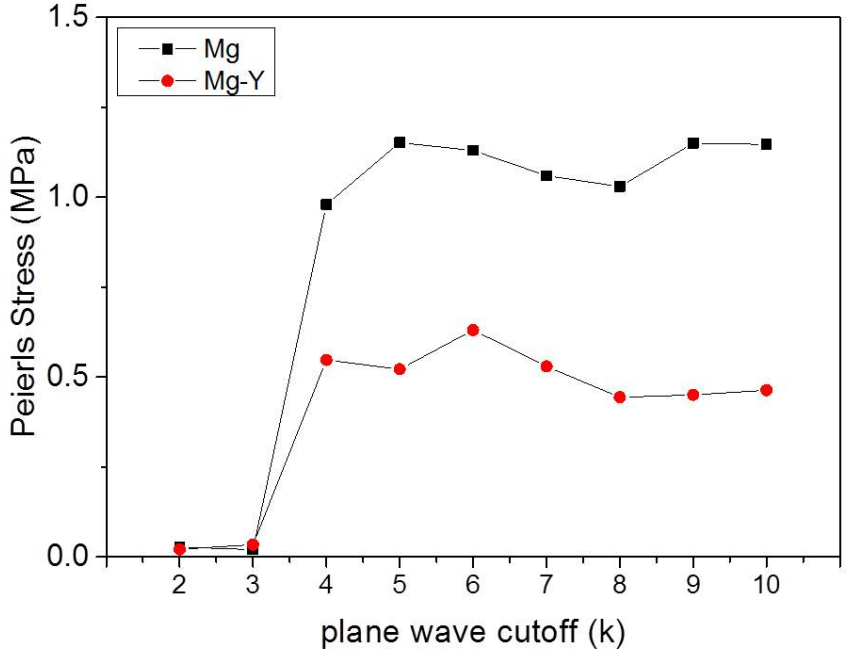
tion [23, 24, 25]. The details and performance of the algorithm are described in Sections 3 and 4

In our previous study [7], we focused on $\langle a\rangle$-type edge dislocations in $\mathrm{Mg}$ alloys that are known to dissociate into two partials separated by wide stacking faults [5, 7]. This type of dislocations can be well described by only four arctan functions, i.e., two for the edge component and two for the screw component. The misfit functions Eq. 2 are then written as follows

$$
\begin{aligned}
& u_{x}(x)=\frac{b}{2 \pi} \arctan \frac{x-d_{e} / 2}{w_{e}}+\frac{b}{2 \pi} \arctan \frac{x+d_{e} / 2}{w_{e}}+\frac{b}{2} ; \\
& u_{z}(x)=\frac{\sqrt{3} b}{6 \pi} \arctan \frac{x-d_{s} / 2}{w_{s}}-\frac{\sqrt{3} b}{6 \pi} \arctan \frac{x+d_{s} / 2}{w_{s}} .
\end{aligned}
$$

where $u_{x}(x)$ and $u_{z}(x)$ describe the edge and the screw displacements, respectively. Here $d_{e, 1}=d_{e, 2}=d_{e} / 2, d_{s, 1}=d_{s, 2}=d_{s} / 2$, $w_{e, 1}=w_{e, 2}=w_{e}$ and $w_{s, 1}=w_{s, 2}=w_{s}$. The weights $A_{1}=A_{2}=\frac{b}{2 \pi}, B_{1}=-B_{2}=\frac{\sqrt{3} b}{6 \pi}$. In the expression of Eq. 3 , the distance $r_{e, 12}=d_{e}$ applies for edge components and $r_{s, 12}=d_{s}$ for screw components. The solution of $\vec{u}$ is illustrated in Figure 1. The two partial dislocations are separated by 7 times of the Burgers vector, which is characterized by the peaks and valley of the misfit density functions $\rho_{x}$ and $\rho_{z}$. A detailed discussion is referred to [7].

\subsection{Five-energy-point Method}

Up to now we have not discussed how to calculate the gamma surface $\gamma$ in the framework of Peierls-Nabarro model. In the two-dimensional case, it can be accurately calculated by applying a dense mesh in the faulted plane within a minimum period of area [26, 7]. This is computationally expensive and unnecessary in some case. We test the convergence of Peierls stress with the cutoff of employed plane waves $k$ in describing the basal-plane gamma surface in $\mathrm{Mg}$ (see Figure 2 ) and found that only five data points are required to obtain a reliable Peierls minima in the solution space and identify the optimal solu- 
stress. The expression of five-energy-point gamma surface is

$$
\begin{aligned}
& \gamma_{5-\text { point }}\left(u_{x}, u_{z}\right)=R_{0} \\
& +R_{1}\left\{\cos \left(2 \pi\left(\frac{u_{x}}{a}-\frac{u_{z}}{\sqrt{3} a}\right)\right)+\cos \left(2 \pi\left(\frac{u_{x}}{a}+\frac{u_{z}}{\sqrt{3} a}\right)\right)+\cos \left(4 \pi \frac{u_{z}}{\sqrt{3} a}\right)\right\} \\
& +R_{2}\left\{\cos \left(2 \pi\left(\frac{u_{x}}{a}-\frac{3 u_{z}}{\sqrt{3} a}\right)\right)+\cos \left(2 \pi\left(\frac{u_{x}}{a}+\frac{3 u_{z}}{\sqrt{3} a}\right)\right)+\cos \left(4 \pi \frac{u_{x}}{a}\right)\right\} \\
& +R_{3}\left\{\cos \left(4 \pi\left(\frac{u_{x}}{a}+\frac{u_{z}}{\sqrt{3} a}\right)\right)+\cos \left(4 \pi\left(\frac{u_{x}}{a}-\frac{u_{z}}{\sqrt{3} a}\right)\right)+\cos \left(8 \pi \frac{u_{z}}{\sqrt{3} a}\right)\right\} \\
& +I_{1}\left\{\sin \left(2 \pi\left(\frac{u_{x}}{a}-\frac{u_{z}}{\sqrt{3} a}\right)\right)-\sin \left(2 \pi\left(\frac{u_{x}}{a}+\frac{u_{z}}{\sqrt{3} a}\right)\right)+\sin \left(4 \pi \frac{u_{z}}{\sqrt{3} a}\right)\right\} \\
& +I_{2}\left\{\sin \left(4 \pi\left(\frac{u_{x}}{a}-\frac{u_{z}}{\sqrt{3} a}\right)\right)-\sin \left(4 \pi\left(\frac{u_{x}}{a}+\frac{u_{z}}{\sqrt{3} a}\right)\right)+\sin \left(8 \pi \frac{u_{z}}{\sqrt{3} a}\right)\right\}
\end{aligned}
$$

where $a$ is the lattice parameter, and $R_{i}(i=0, \ldots, 3), I_{i}(i=$

expression one group of sin-type functions $\left(I_{3}\right)$ and all the rest terms (i.e., the higher frequency terms) are dropped since their coefficients are ignorable $\left(<10^{-2} \mathrm{mJm}^{-2}\right.$ in $\mathrm{Mg}$, for example).In Eq 7 there are six coefficients that needs to be determined. In principle, if six data points on the gamma surface are known, we can determine the coefficients by solving a group of equations. By default, the gamma surface always goes through zero point, i.e., $\gamma(0,0)=0$, so we only need five data points in total.

This expression is applicable for basal dislocations in all hcp crystals and dislocations on the $\{111\}$ plane in all fcc crystals. The Peierls stress in $\mathrm{Mg}$ is very low (i.e., $0.5-0.8 \mathrm{MPa}$ [28, 29, [30]), so it requires a very accurate gamma surface as input of 175 the model. If the information of interest is the dislocation core, less terms than five are enough [7]. Also for dislocations with large Peierls stress the requirement of gamma-surface accuracy may also lower down.

As an example, we study the $\{111\} \frac{1}{2}\langle 1 \overline{1} 0\rangle$ in FeNiCoCrMn high-entropy alloy. Even though the $\{111\}$ stacking is differ-180 ent from $\{0001\}$, their in-plane symmetric operations are same. Therefore our symmetrized-plane-wave expression for gamma surface (Eq7) applies to this dislocation in the high-entropy alloy. We take only two energy-points (i.e., the intrinsic and globally unstable stacking fault energies $\gamma_{i s f}$ and $\gamma_{u s}$ from [27]) $)_{185}$ as input of our model. Inserting the two stacking fault energies into Eq. 7. we have $R_{0}=69 \mathrm{mJm}^{-2}, R_{1}=33 \mathrm{mJm}^{-2}$ and $I_{1}=-24 \mathrm{mJm}^{-2}$. With trial solution in the form of Eq. 6 we determine the values for all the involved parameters (see Table 2). The dislocation core of the high-entropy alloy described by ${ }_{190}$ these parameters are shown in Figure 3 Due to the existence of the stable stacking fault and its low energy, the dislocation dissociates into two Shockley partial dislocations. Interestingly, the stacking fault width of about $3 b$ is only half of that of the $\mathrm{I}_{1}$ stacking fault in $\mathrm{Mg}$ even though its stacking fault energy ${ }_{195}$ is only half of the latter. Based on the dislocation core structure the Peierls stress is computed to be $168.4 \mathrm{MPa}$, which is in good agreement with experimentally measured Peierls stress $175 \pm 5 \mathrm{MPa}$ and previously predicted one $178 \mathrm{MPa}$ also using Peiers-Nabarro model [27].

For dislocations that do not dissociate, one-dimensional

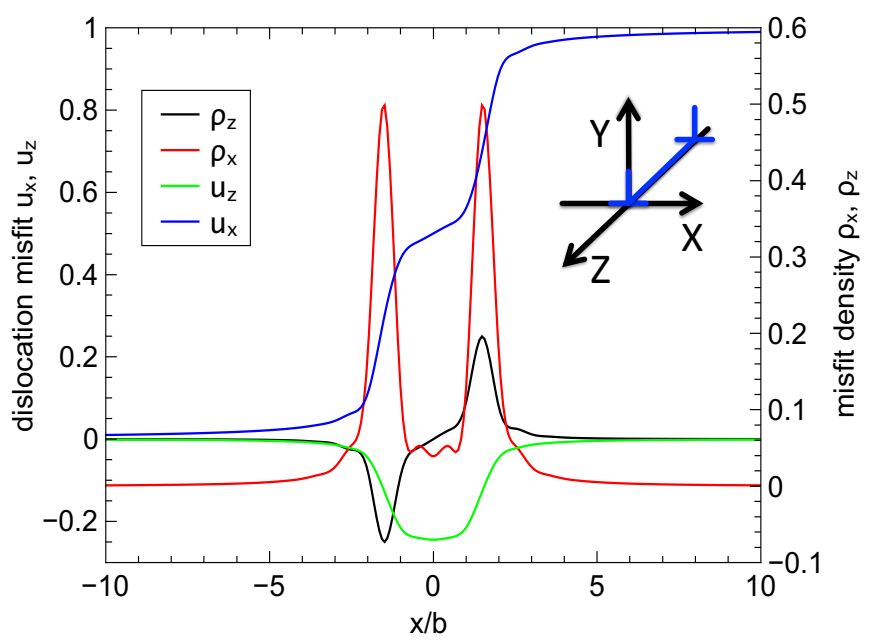

Figure 3: The two components $\left(u_{x}, u_{z}\right)$ of the misfit function of $\{111\} \frac{1}{2}\langle 1 \overline{1} 0\rangle$ dislocation in $\mathrm{CrFeCoNiMn}$ high entropy alloy and their derivatives $\left(\rho_{x}, \rho_{z}\right)$. The dislocation is placed in a coordinate as shown in the inset, i.e., the dislocation line runs along $z$ axis, and the slip plane is $x z$ plane. The $z$ axis is perpendicular to the paper.

the GSFE curve is the minimum energy path on the gamma surface, and the left part of the gamma surface will not be accessed even though a full gamma surface is available. Such an example for the prismatic dislocation in $\mathrm{Mg}$ can be found in [31].

\section{Particle Swarm Optimization}

The algorithm of Particle Swarm Optimization (PSO) simulates the social behaviors of organisms, e.g., fishes, bees, birds, etc [23, 24, 25]. In our everyday life we have such an impression that a swarm of bees can always find their flower source. Suppose each bee represents a possible solution (i.e. a particle in solution space), the flower source represents then the globally optimal solution. In principle, given enough time the particles can always find a locally or the globally optimized solution. The probability to find the global one becomes higher by increasing the size of the swarm. During the time to the flower source, bees have a globally consistent behavior, so they must communicate and exchange their "feeling" for the direction of the flower source. This group behavior can effectively avoid bees to fly in wrong directions (local minima in solution space).

The past decade has seen a quick development of PSO and different variants of the original algorithm have emerged [32]. The details of the PSO algorithm employed in this study are described in Table 3 Each particle $i$ of a swarm of size $N$ is a point in the multidimensional position space (characterized by dimension number $n$ ). Initially the position of each particle is assigned random numbers within the safe bounds $\left(b_{\text {lo }}, b_{\text {up }}\right)$ that covers the globally optimal solution. In the following steps, a particle is led by its own "feeling" of the optimal solution (i.e. its current local best position) and also by the that of the group (i.e. the current global best position) through communication. Therefore, the local and global best positions need to be computed at initial and the following time step. At the first 
Table 2: The values of input and output parameters for our Peierls-Nabarro modeling of $\{111\} \frac{1}{2}\langle 1 \overline{1} 0\rangle$ dislocation in FeNiCoCrMn high-entropy alloy. The calculated Peierls stress by us and previous experimental and theoretical results [27] are listed as well. In our calculation the Poisson ratio $v=0.3$.

\begin{tabular}{lllllll}
\hline \hline Material & $a_{0}(\AA)$ & Burger vector $\vec{b}$ & $K_{e}(\mathrm{GPa})$ & $\gamma_{u s}\left(\mathrm{mJm}^{-2}\right)$ & $\gamma_{i s f}\left(\mathrm{mJm}^{-2}\right)$ & $\tau_{p}(\mathrm{MPa})(\mathrm{previous})$ \\
FeNiCoCrMn & $3.59[27]$ & $\frac{\sqrt{2}}{2} a_{0}\langle 110\rangle$ & $84[27]$ & $192[27]$ & $17[27]$ & $175 \pm 5[27]($ Experiment, 77K), 178 [27](Theory, 0K) \\
\hline$A_{1}\left(=A_{2}\right)$ & $B_{1}\left(=-B_{2}\right)$ & $d_{e} / b$ & $d_{s} / b$ & $w_{e} / b$ & $w_{s} / b$ & $\tau_{p}(\mathrm{MPa})($ this study, 0K) \\
0.5 & 1.0 & 3.12 & 2.94 & 0.31 & 0.36 & 168.4 \\
\hline \hline
\end{tabular}

time step, the initial positions are also their best local positions. By comparing these initial positions we can find the initial best global position. The initial velocity of each particle is also assigned as a random number in the range $U\left(-\left|b_{\mathrm{up}}-b_{\mathrm{lo}}\right|,\left|b_{\mathrm{up}}-b_{\mathrm{lo}}\right|\right)$ or a subset of it.

At the next time step, the initial velocities $v_{i, d}$ are updated firstly by

$$
v_{i, d} \leftarrow \omega v_{i, d}+\phi_{p} r_{p}\left(p_{i, d}-x_{i, d}\right)+\phi_{g} r_{g}\left(g_{d}-x_{i, d}\right)
$$

where $x_{i, d}$ is the $d^{\text {th }}$ dimension of the $i^{\text {th }}$ particle, $g$ and $p$ are the global and local best positions. The second term on the right e particle is guided by its own sense and the third term describes the particle is affected by its surrounding particles through communication. The weight parameters $\phi_{p}$ and $\phi_{g}$ determine how the particles' velocities are affected by their local and global "feelings". To increase the randomness in updatThe constant $\omega$ can be optimized to improve the efficiency of the algorithm. An optimized $\omega$ is close to 1 and 1 is a good choice in most of the case. With updated velocities the new positions are calculated by $x_{i} \leftarrow x_{i}+v_{i}$. At the new position the aim function for each particle is then calculated and compared with that of its old position. Accept the new position if the new local best position is in the wanted direction, i.e., $\mathrm{a}_{245}$ direction close to the gradient of the aim function. The last step is to update the global best position in the same procedure as in the initial time step. The velocities are updated using Eq 8 These steps are iterated until the value of the aim function meets the termination criterion.

Since the time-consuming part of the algorithm (line 8-20 in Table 3 consists of two for-loops, such a structure is par230 ticularly suitable to be parallelized. Inside this part computing the values of aim function $f$ can also be computationally expensive, which is exactly the case for the calculation of $E_{c}$ in $_{255}$ Peierls-Nabarro model. Fortunately this procedure also consists of a for-loop that is not difficult to be parallelized. It is worth to notice that the PSO requires the particles to exchange their "feelings" for the optimal solution, so an ideal scaling of speedup is not expected.

\section{Scaling and Performance}

The algorithm of PSO is implemented in $\mathrm{C}++$ language on the GenuineIntel machine of "CMMC" cluster of Max-Planck-265 Institut für Eisenforschung $\mathrm{GmbH}$. The cluster consists of 650 nodes and for each node there are 20 shared-memory cores. As an initial test, we parallelize the algorithm using OpenMP library for shared-memory cores. As an example, we use the
Table 3: (Algorithm 2) The Algorithm of Particle Swarm Optimization. It is readily used to solve the Peierls-Nabarro equation when the aim function $f \leftarrow E_{c}$ and a particle's position $p_{i} \leftarrow\left(w_{i}, A_{i}, B_{i}, r_{i j}\right)$.

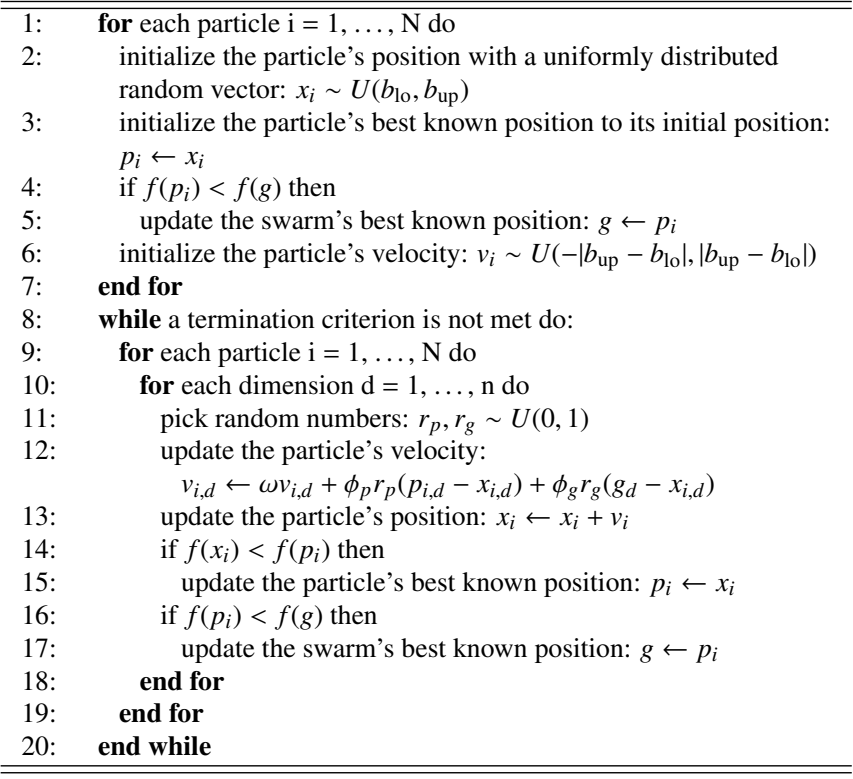

algorithm to solve the Peierls-Nabarro equation to obtain the core structure of $\{10 \overline{1} 0\}\langle 11 \overline{2} 0\rangle$ dislocation. We test for 30,300 and 3000 particles and 1000, 10000 and 100000 iteration steps.

The Performance of the parallelized algorithm is shown in Figure 4(a). The best performance is obtained for 3000 particles and 100000 iteration steps, i.e.,77.5\%. The Speedup scales linearly with the number of cores, which demonstrates that the PSO is very suitable for future massive parallelization.

Figure 4(b) shows the performance is also affected by problem size/workload, i.e., the numbers of iteration steps and particles in this case. In order to separately check how the speedup changes with the number of iteration step and particle size, we keep one of them as a constant. The performance of the tests is obtained with 20 cores. When the number of iteration step is 10000 , the speedup increases from 10.5 to 15.5 with more particles; when the particle size is 300 , the speedup changes from 12.2 to 15.4 with more iteration steps. However, the gain with heavier workload converges quickly with the numbers of particles and iteration steps. For example, when the number of particles increases 10 times from 300 to 3000 , the speedup changes only from 15 to 15.5 . The underlying reason lies in the principle of parallelization, which is summarized in Amdahl's law. Amdahl's law can be formulated as the speedup $S=\frac{1}{1-p+p / N}$, where $\mathrm{p}$ is the parallelable part of a job, $N$ is the number of cores applied in one simulation. According to the law, the ac- 


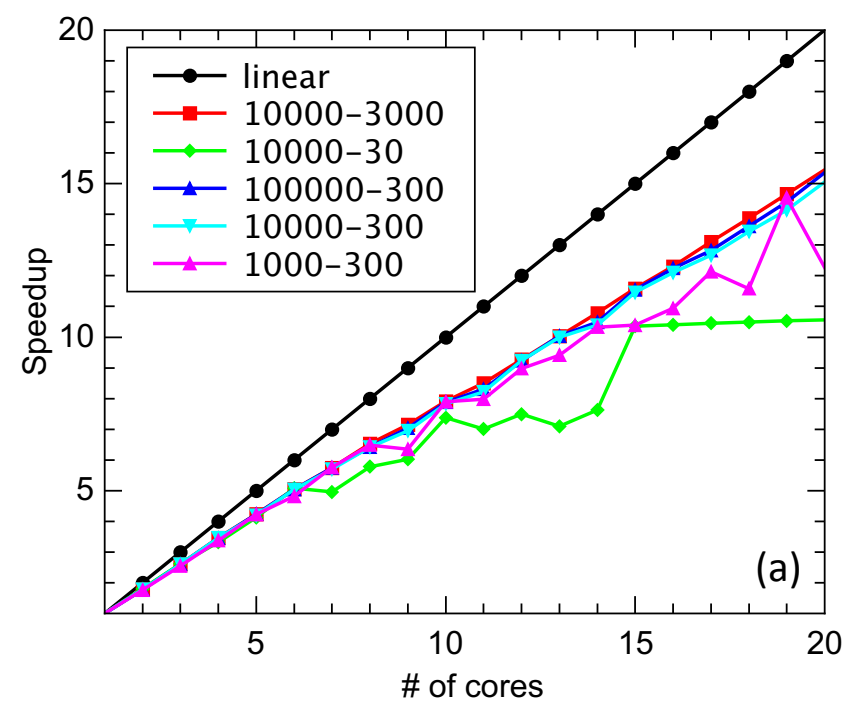

\# of particles

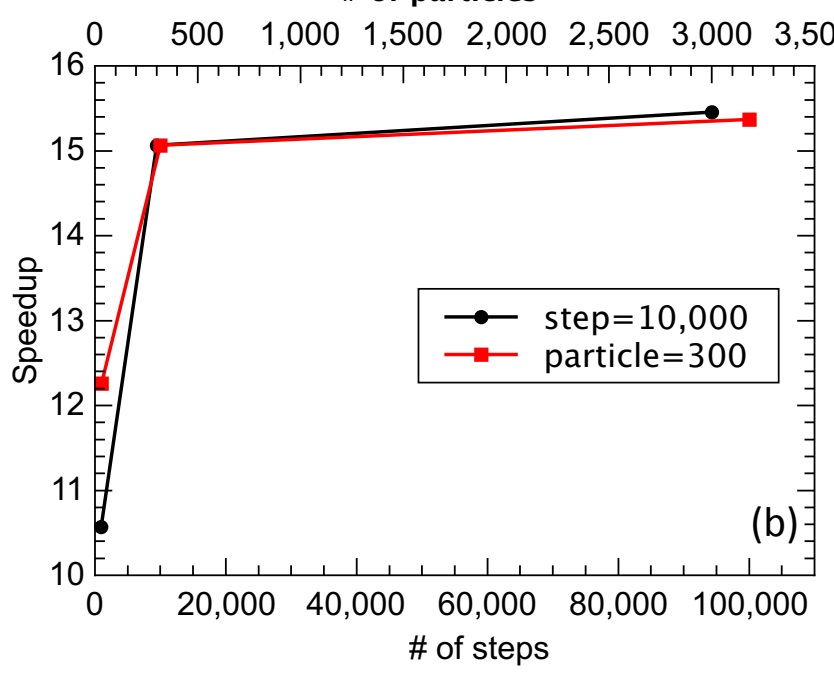

Figure 4: The performance of our accelerated Particle Swarm Algorithm (PSA) (a) The speedups of different problem sizes increase with applied core number. (b) The speedups with 20 cores increase with problem sizes (number of particles or iteration steps).

celeration is gained by distributing the parallelable part of one job to multiple cores and computing them in a parallel man-ззо ner. Since there is always serial part of one task $(p<1)$, the best performance $S_{\max }$ is not $N$ but $\frac{1}{1-p}$. The law also clearly shows that the performance can be enhanced by increasing the the Peierls-Nabarro model.

\section{Acknowledgment}

Research sponsored by the Laboratory Directed Research and Development Program of Oak Ridge National Laboratory, 290 managed by UT-Battelle, LLC, for the U. S. Department of Energy. This research used resources of the Oak Ridge Leadership Computing Facility, which is supported by the Office of Science of the U.S. Department of Energy under contract no. DE-AC0500OR22725. Z.P. also acknowledges the financial support from Planck-Institut für Eisenforschung $\mathrm{GmbH}$ and the computing resources of the institute.

\section{References}

[1] P. Hirth, J. Lothe, Theory of Dislocations, 2nd Edition, Elsevier Ltd, 1982.

[2] D. Hull, D. Bacon, Introduction to Dislocations, 5th Edition, New York Wiley \& Sons, 2011.

[3] D. J. Bacon, J. W. Martin, The atomic structure of dislocations in h.c.p. metals i. potentials and unstressed crystals Philosophical Maga-

zine A 43 (4) (1981) 883-900 arXiv : http://dx doi org/10.1080/ 01418618108239498 doi:10.1080/01418618108239498 URL http://dx.doi.org/10.1080/01418618108239498

[4] T. Nogaret, W. Curtin, J. Yasi, L. H. Jr, D. Trinkle, Acta Mater. 58 (13) (2010) $4332-4343$.

5] J. A. Yasi, T. Nogaret, D. R. Trinkle, Y. Qi, L. G. H. Jr, W. A. Curtin, Modelling and Simulation in Materials Science and Engineering 17 (5) (2009) 055012

[6] I. Shin, E. Carter, Modelling and Simulation in Materials Science and Engineering 20 (1) (2012) 015006.

7] Z. Pei, D. Ma, M. Friák, B. Svendsen, D. Raabe, J. Neugebauer, Phys. Rev. B 92 (2015) 064107.

[8] D. R. Trinkle, C. Woodward, The chemistry of deformation: How solutes soften pure metals Science 310 (5754) (2005) 1665-1667. arXiv:http://science.sciencemag.org/content/310/5754/

[9] G. Leyson, L. H. Jr., W. Curtin, Acta Mater. 60 (13-14) (2012) 5197 5203.

With the parallelized algorithm of PSO we can solve the Peierls-Nabarro equation within several minutes for dislocations in conventional alloys. Since there are usually more than five components in high-entropy alloys, it is expected there $\mathrm{ex}^{-340}$ ist more local minima in the search space for solutions. Therefore, for dislocations in high-entropy alloys more particles and iteration steps are probably required to solve the equation, which means a higher computational cost and longer run time. ${ }^{345}$ In this case our parallelized algorithm is particularly useful in reducing the run time and increasing the efficiency of solving
[10] I. Shin, E. A. Carter, International Journal of Plasticity 60 (0) (2014) 58 70.

[11] J. A. Yasi, L. G. H. Jr., D. R. Trinkle, Acta Mater. 58 (17) (2010) 5704 5713 .

[12] J. A. Yasi, L. G. H. Jr., D. R. Trinkle, Acta Mater. 60 (5) (2012) 2350 2358.

[13] J. E. Sinclair, P. C. Gehlen, R. G. Hoagland, J. P. Hirth, J. Appl. Phys. 49 (1978) 3890.

[14] C. Woodward, S. I. Rao, Phys. Rev. Lett. 88 (2002) 216402.

[15] R. Peierls, The size of a dislocation Proceedings of the Physical Society 52 (1) (1940) 34. URL http://stacks . iop.org/0959-5309/52/i=1/a=305

[16] F. R. N. Nabarro, Dislocations in a simple cubic lattice Proceedings of the Physical Society 59 (2) (1947) 256 URL http://stacks . iop.org/0959-5309/59/i=2/a=309

[17] G. Schoeck, Materials Science and Engineering: A 400-401 (0) (2005) 7 -17 .

[18] G. Leibfried, H. Dietze, Z. Physik 131 (1951) 113.

[19] V. Vítek, Phil. Mag. 154 (18) (1968) 773-786.

[20] S. F. Wang, Journal of Physics A: Mathematical and Theoretical 42 (2) (2009) 025208.

[21] V. V. Bulatov, E. Kaxiras, Phys. Rev. Lett. 78 (1997) 4221-4224.

[22] G. Lu, N. Kioussis, V. V. Bulatov, E. Kaxiras, Materials Science and Engineering: A 309310 (0) (2001) $142-147$. 
[23] J. Kennedy, R. Eberhart, Particle swarm optimization, IEEE (1995) 1942.

[24] R. Poli, Journal of Artificial Evolution and Applications 2008 (2008) 1 10.

[25] D. Ma, M. Friák, J. von Pezold, D. Raabe, J. Neugebauer, Acta Mater. 85 (0) (2015) $53-66$.

[26] Z. Pei, L.-F. Zhu, M. Friák, S. Sandlöbes, J. v. Pezold, H. Sheng, C. Race, S. Zaefferer, B. Svendsen, D. Raabe, J. Neugebauer, New J. Phys. 15 (4) (2013) 043020

[27] L. Patriarca, A. Ojha, H. Sehitoglu, Y. Chumlyakov, Slip nucleation in single crystal fenicocrmn high entropy alloy Scripta Materialia 112

1) (2016) 54-57.doi:http://dx.doi.org/10.1016/j.scriptamat.

$360 \quad 2015.09 .009$

11 URL http://www.sciencedirect.com/science/article/pii/ S1359646215003991

[28] E. Schmid, Z. Elektrochem 37 (1931) 447.

[29] P. Bakarian, C. Mathewson, Trans. AIME 152 (1943) 226.

365 [30] H. Conrad, W. Robertson, Trans. AMIE 209 (1957) 503.

[31] T. Tsuru, Y. Udagawa, M. Yamaguchi, M. Itakura, H. Kaburaki, Y. Kaji, Journal of Physics: Condensed Matter 25 (2) (2013) 022202.

[32] F. F. de Vega, J. I. H. Pérez, J. Lanchares, Parallel Architectures and Bioinspired Algorithms, Springer-Verlag Berlin Heidelberg, 2012. 